\title{
Enfermedad de Fabry. Manejo anestésico en trasplante renal, a propósito de un caso
}

\author{
Fabry disease. Anesthetic management in kidney transplant, \\ report of case
}

Pedro Luis Quishpe Pila ${ }^{1}$, Andrea Carolina Pico Aguilar ${ }^{1}$

\begin{abstract}
Introduction: Fabry disease (FD) also known as Anderson Fabry disease is a rare disorder linked to the $X$ chromosome, which produces mutations in the coding of the GLA gene involved in the production of the enzyme $\alpha$-galactosidase A, whose complete or partial deficiency leads to the intracellular accumulation of globotriaosylceramide and glycosphingolipids. Clinical case: We present the case of a 39 year old female patient admitted to hospital with a diagnosis of terminal chronic kidney disease of 8 years of evolution as a possible cause of nephropathy, Fabry disease diagnosed in a patient, after detailed studies, kidney transplantation is considered for improvement of your lifestyle. Discussion: Patients with Fabry disease should be considered as high risk surgical and anesthetic should have a strict assessment and evaluation of cardiovascular and respiratory function, to anticipate the complications associated with reperfusion of the transplanted organ. Conclusion: The use of balanced or intravenous modality has been described among the anesthetic possibilities without reaching a consensus so far, however the two modalities can be used and their analgesic management can be performed with plexus blocks or regional anesthesia.
\end{abstract}

\section{RESUMEN}

Introducción: La enfermedad de Fabry (FD) también conocida como enfermedad de Anderson Fabry es un trastorno raro ligado al cromosoma $X$, que produce mutaciones en la codificación del gen GLA partícipe en la producción de la enzima $\alpha$-galactosidasa A, cuya deficiencia completa o parcial conduce a la acumulación intracelular de globotriaosilceramida y glicosfingolípidos.

\section{Key words:}

Chronic kidney disease, Fabry disease, kidney transplant, lysosomal storage disease

\section{Palabras clave:}

Enfermedad renal crónica, Enfermedad de Fabry, trasplante renal, enfermedad de depósito lisosomal

Residente del Posgrado de Anestesiología de la Universidad San Francisco de Quito. Hospital Carlos Andrade Marín.

Fecha de recepción: 12 de enero de 2019

Fecha de aceptación: 16 de febrero de 2019

\section{ORCID}

https://orcid.org/0000-0002-6406-6987

Correspondencia:

Pedro Luis Quishpe Pila

Email: pedro_quishpe@yahoo.es/pedroq409@gmail.com 
Caso clínico: Se presenta el caso de una paciente femenina de 39 años de edad ingresada a hospitalización con diagnóstico de enfermedad renal crónica terminal de 8 años de evolución como posible causa de nefropatía, enfermedad de Fabry diagnosticada en paciente, tras estudios detallado se considera trasplante renal para mejora de su estilo de vida. Discusión: Los pacientes con enfermedad de Fabry deben ser considerados como de alto riesgo quirúrgico y anestésico, deben contar con una estricta valoración y evaluación sobre la función cardiovascular y respiratoria, para así preveer las complicaciones asociadas a la reperfusión del órgano trasplantado. Conclusión: Se han descrito entre las posibilidades anestésicas el uso de modalidad balanceada o intravenosa sin llegar aún a un consenso hasta el momento, sin embargo, las dos modalidades pueden ser utilizadas y su manejo analgésico se puede realizar con bloqueos del plexo o anestesia regional.

\section{Introducción}

- I caso describe una entidad rara denominada - como enfermedad de Fabry (FD) también cono- cida como enfermedad de Anderson Fabry este es un trastorno raro ligado al cromosoma X[1],[2], que produce mutaciones en la codificación del gen GLA partícipe en la producción de la enzima $\alpha$-galactosidasa A, cuya deficiencia completa o parcial conduce a la acumulación intracelular de globotriaosilceramida (Gb3) y otros glicosfingolípidos[3]-[5], es decir, un trastorno innato del metabolismo, que conduce a la acumulación de globotriaosilceramida (Gb3) dentro de los lisosomas en una amplia variedad de células, incluida endotelial, neuronales, renal, cardíaca y de la raíz dorsal[6]-[10].

El manejo anestésico en trasplante renal tiene particularidades en vista que es uno de los grupos con mayor morbilidad, una valoración preoperatoria adecuada, historia clínica anestésica detallada, horas posteriores a diálisis, peso seco y contar con exámenes de laboratorio actualizados en especial perfil de electrolitos por posibles alteraciones durante el transanestésico[6],[11]-[13], determinación de factores de riesgos y estado del paciente. Dentro de la evaluación preoperatoria hay que tomar en cuenta la estatificación de riesgo quirúrgico anestésico y capacidad funcional (NYHA y METS), valoraciones multidisciplinarias, obtención de consentimiento informado y la necesidad de manejo de dolor posterior a trasplante se han visto varias dificultades en relación a la utilidad de catéteres epidurales por el incremento de riesgos en relación a la inmunosupresión, sin embargo, no hay consenso que impida el manejo de dispositivos intratecales para manejo de dolor, el procedimiento quirúrgico requiere comunicación con el equipo de trabajo[14]-[18].

\section{Caso clínico}

Se trata de una paciente femenina de 39 años de edad, con diagnóstico de enfermedad renal crónica terminal estadio 5, en hemodiálisis filtración programado de forma emergente para trasplante renal de donante cadavérico entre sus antecedentes personales HTA, enfermedad de Fabry, sin alergias, insuficiencia renal crónica anúrica patología de 8 años de evolución, como etiología probable depósitos lisosomales en células renales, exámenes preoperatorios que demuestran hemoglobina 10, con 2 horas pos diálisis, peso seco $55 \mathrm{~kg}$, presión arterial controlada con calcioantagosnista y ARA II, en espera para trasplante renal, en seguimiento por endocrinología actual sin contraindicación, mantiene esquema en manejo de proteinuria y posibilidad de manejo enzimático pos trasplante.

Al examen físico el paciente, estable, con presencia de fístula AV en extremidad superior izquierda, trill presente. Test de Allen normal en las dos extremidades superiores. Espirometría dentro de parámetros adecuados $\mathrm{FEV}, 82 \%$, azoados pos diálisis creatinina 2,3 electrolitos potasio 4,3. Ecocardiograma normofunción sistólica diastólica de VI con hipertrofia concéntrica de VI. Se lo cataloga como riesgos quirúrgico alto, riesgo de sangrado CAPRINI alto, equivalentes metabólicos METS 4, capacidad funcional NYHA II, riesgo anestésico ASA IV. Posibilidad de complicaciones pulmonares $3 \%$.

\section{Manejo intraoperatorio}

Paciente en sala de operaciones se procede a monitoreo no invasivo e invasivo (Figura 1) se coloca catéter peridural a nivel de I2-13 con técnica aséptica y pérdida de resistencia, comprobación de catéter con 


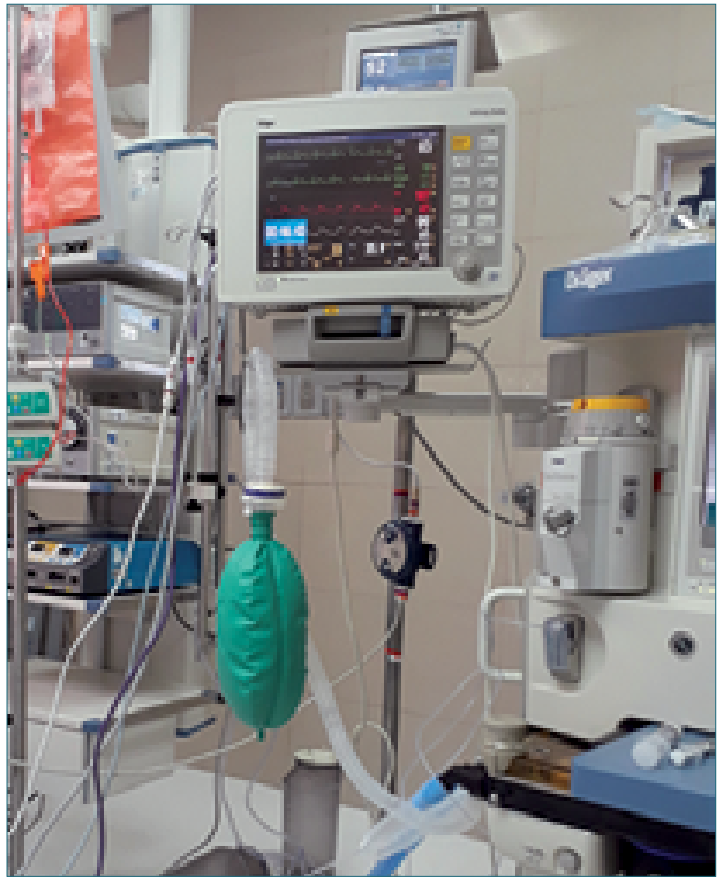

Figura 1. Monitoreo hemodinámico continuo de paciente sometido a trasplante renal (FC; PANI; TAI; BISS; TOFF). Foto obtenida por el autor. *HCAM, *Hospital Carlos Andrade Marín.

solución lidocaína con 15 ug de epinefrina, negativo cambios hemodinámicos, extremidad superior izquierda con presencia de fístula arteriovenoso para hemodiálisis, acceso arterial radial derecho con técnica estéril y previa infiltración con anestésico local (lidocaína 2\%). Inducción infusión de remifentanilo, propofol IV en bolo y relajación neuromuscular con rocuronio, intubación tras TOFF 0, laringoscopia directa convencional, Cormack Lehane G1 se coloca tubo endotraqueal 7.0 con balón.

Mantenimiento anestésico balanceado con $\mathrm{FIO}_{2}$ $50 \%$, sevolfuorane CAM 0,5 y remifentanilo dosis 0,2 a $0,25 \mathrm{ug} / \mathrm{kg} / \mathrm{min}$, se coloca catéter venoso central yugular derecho ecoguiado único intento, durante transanestésico en primera fase (prerenal) mantenimiento balanceado con PAM sobre $80 \mathrm{mmHg}$ independiente de vasopresor administramos antibiótico profilaxis, durante la segunda fase (cirugía de banco) mantenemos esquema balanceado con disminución de infusiones BISS se mantiene en 50 (Figura 1), sin alteraciones hemodinámicas, isquemia fría 3 horas, administración de inmunosupresores infundidos en $30 \mathrm{~min}$. Tercera fase (revascularización) (Figura 2) tras colocación de injerto y pinzamiento arterial necesidad de incremen- to de PAM sobre $100 \mathrm{mmHg}$ con necesidad de vasopresor noradrenalina titulación dosis máxima 0,1 ug/ $\mathrm{kg} / \mathrm{min}$, carga y optimización intravascular con cristaloide lactato ringer antes de revascularización administración de furosemida, con posterior presencia de diúresis en uréter, durante la cuarta fase (tardía) necesidad de mantener PAM sobre $100 \mathrm{mmHg}$ disminución de vasopresor hasta remisión total al despertar hay que recalcar parámetros dinámicos durante intervención VVP de inicio 7 se lo mantiene hasta inicio de 3 Fases, se toman muestras seriales de gasometrías, al inicio de cirugía, clampeo, posclampeo, las mismas que demuestran trastornos ácido base esperadas y corregidas de forma paulatina, mantiene parámetros dinámicos adecuados sin necesidad de administración de hemocomponentes. Diúresis presente al finalizar intervención (Figura 3).

Se decide extubación de paciente tras reversión de bloqueo independiente de dosis de relajante neuromuscular, uso de sugamadex posterior TOFF 100, ventilación espontánea reflejos protectivos de vía aérea presentes PAFI 200 extubación sin contratiempos. Se transfiere a Unidad de Cuidados Intensivos con hemodinamia sostenida, despierta Glasgow 15 sin dolor, se mantiene infusión peridural con adecuado control del mismo. Permanece 2 días en unidad de

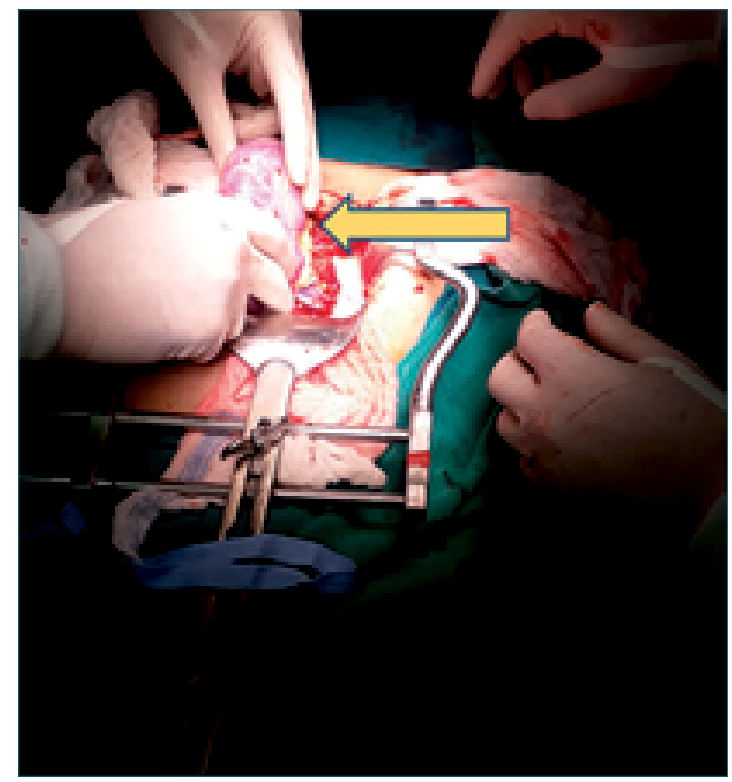

Figura 2. Revascularización del injerto, la flecha indica reperfusión por tonalidad rosado en toda su amplitud. Foto obtenida por el autor. ${ }^{*}$ HCAM, *Hospital Carlos Andrade Marín. 


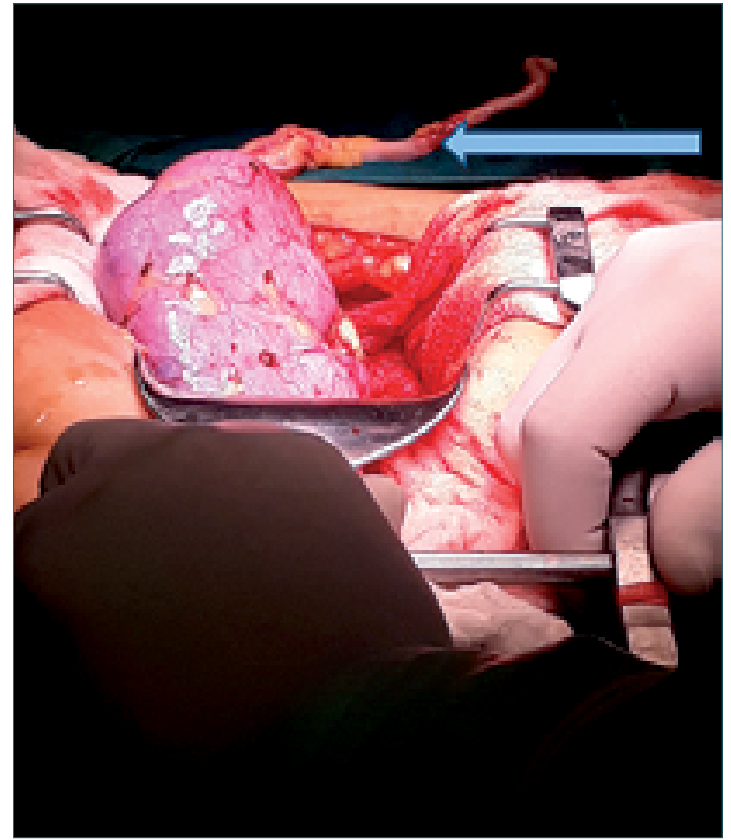

Figura 3. Riñón trasplantado presencia de diuresis, la flecha indica el uréter. Foto obtenida por el autor, *HCAM, *Hospital Carlos Andrade Marín.

cuidados intensivos posterior manejo por nefrología y servicio de trasplantes.

\section{Discusión}

La prevalencia de la enfermedad de Fabry se la estima entre 0,85 y 2,5 casos por 100.000 personas, varían entre el fenotipo severo "clásico" en varones hasta un aparente asintomático observado ocasionalmente en mujeres[1],[4],[6],[12],[19], dentro de las características clínicas incluyen acroparestesia, angioqueratomas, acompañadas de crisis de dolor coincidentes con el caso presentado, sin embargo, no presenta trastornos gastrointestinales, disfunción corneal, lo severo del depósitos lisosomal en células cardiacas, neurológicas y renales conduce a miocardiopatía, insuficiencia renal o enfermedad cerebrovascular de gran importancia en el manejo anestésico[6],[9],[15],[19]-[21], en vista de la necesidad de valoración preoperatoria y anticipo a posibilidades de complicaciones durante el transoperatorio o posquirúrgico inmediato, en nuestro caso la acumulación condujo a insuficiencia renal terminal dicha nefropatía por acumulo progresivos de GL-3 en el parénquima renal en las células tubulares, glomerulares inicio mecanismos de injuria, en un proceso similar a nefropatías proteinurias caracterizadas por producción de óxido nítrico $(\mathrm{ON})$, radicales libres de oxígeno (ROS) y otros mediadores inflamatorios los cuales conducen a disfunción microvascular, oclusión e isquemia[4],[7],[18],[20],[21], de esto la condición de insuficiencia renal crónica se ha considerado como la principal causa de morbilidad en pacientes con enfermedad de Fabry, el trasplante renal debe ser considerado tempranamente en estos pacientes debido a que la evidencia actual demuestra seguridad en el procedimiento y mayor supervivencia en relación a los pacientes con enfermedad de Fabry que permanecen en diálisis[7],[9],[15],[17]. El objetivo del trasplante renal fue mejora en la calidad de vida en nuestra paciente, se consideró funcionalidad del injerto a largo plazo, evitando exacerbación de comorbilidades, no se encontró contraindicaciones para trasplante entre las cuales destaca edad, estado físico o patologías infecciosas, la evaluación cardiovascular pretrasplante demostró hipertrofia ventricular izquierda sin signos de falla cardiaca, por lo que se consideraron pacientes de alto riesgo cardiovascular; por todo lo antedicho el paciente con enfermedad de Fabry debe ser considerado como paciente de alto riesgo quirúrgico y anestésico la estricta valoración preanestésica con evaluación sobre la función cardiovascular y respiratoria, deben contar con estudios detallados, ecocardiograma, espirometria, hemograma y perfil bioquímico completo[9],[10],[18],[20]. No existe diferencia en los protocolos anestésicos utilizados de forma rutinaria para trasplantes renales la consideración del monitoreo hemodinámico estricto debe ser usada, además, incluir medidas invasivas para monitoreo, en relación al manejo del dolor se debería evitar interacciones medicamentosas con analgésicos intravenosos e inmunosupresores por lo que anestesia regional con catéteres epidurales se recomienda, debe evitarse la participación de la inhibición de la molécula M-TOR debido a la autofagia ya alteración del injerto[9].

\section{Conclusiones}

La enfermedad de Fabry es una patología rara asociada al cromosoma $X$, cuyo desenlace con nefropatía es una causa importante de morbilidad, especialmente en pacientes sometidos a TSR, el manejo anestésico consiste en proveer estabilidad hemodinámica, valiéndose de un monitoreo invasivo y no invasivo.

El trasplante renal en un paciente afecto con enfermedad de Fabry se considera adecuado en cuanto mejora la calidad de vida, nuestra labor como anes- 
tesiólogos es brindar un adecuado campo quirúrgico adecuado, garantizando perfusión y evitando las ma- yores complicaciones como lo son cardiovasculares y respiratorias.

\section{Referencias}

1. Nishioka R, Sofue T, Moritoki M, Nishijima Y, Nishioka S, Hara T, et al. [Case Report: A case of living-donor kidney transplantation from a heterozygote mother to a hemizygote son of Fabry disease diagnosed by donated allograft biopsy]. Nippon Naika Gakkai Zasshi. 2015 Apr;104(4):775-80. https://doi. org/10.2169/naika.104.775 PMID:26536741

2. Tran Ba SN, Lidove O, Dorent $R$, Debauchez $M$, Nataf $P$, Delahousse $M$, et al. [Combined heart and kidney transplantation in Fabry's disease: long-term outcomes in two patients]. Rev Med Interne. 2017 Feb;38(2):13742. https://doi.org/10.1016/j. revmed.2016.03.008 PMID:27241078

3. Cabrera G, Perretta F. Fabry disease. A potential pitfall $A$ family with a novel intronic mutation. Mol Genet Metab Rep. 2018 Sep;17:16-7. https://doi.org/10.1016/j. ymgmr.2018.07.001 PMID:30211005

4. Del Pino $M$, Andrés $A$, Bernabéu AÁ, de Juan-Rivera J, Fernández E, de Dios García Díaz J, et al. Fabry Nephropathy: An Evidence-Based Narrative Review. Kidney Blood Press Res. 2018;43(2):406-21. https:// doi.org/10.1159/000488121 PMID:29558749

5. Sharma A, Sartori M, Zaragoza JJ, Villa G, Lu R, Faggiana E, et al. Fabry's disease: an example of cardiorenal syndrome type 5. Heart Fail Rev. 2015
Nov;20(6):689-708. https://doi. org/10.1007/s10741-015-9500O PMID:26232292

6. Villa G, Romagnoli S, Sharma A, Ronco C. [Fabry's disease: an example of cardiorenal syndrome type 5]. G Ital Nefrol. 2017 Mar;34 Suppl 69:131-41. PMID:28682034

7. Wani MM, Khan I, Bhat RA, Ahmad M. Fabry's Disease: Case Series and Review of Literature. Ann Med Health Sci Res. 2016 May-Jun;6(3):193-7. https://doi. org/10.4103/2141-9248.183935 PMID:27398254

8. Lenders M, Oder D, Nowak A, Canaan-Kühl S, Arash-Kaps L, Drechsler C, et al. Impact of immunosuppressive therapy on therapy-neutralizing antibodies in transplanted patients with Fabry disease. J Intern Med. 2017 Sep;282(3):241-53. https:// doi.org/10.1111/joim.12647 PMID:28682471

9. Capelli I, Battaglino G, Baraldi O, Ravaioli M, Cuna V, Moretti I, et al. [Kidney Transplantation and inborn errors of metabolism]. G Ital Nefrol. 2015;32 Suppl 64. PMID: 26479053

10. Odani K, Okumi M, Honda K, Ishida H, Tanabe K. Kidney transplantation from a mother with unrecognized Fabry disease to her son with low $\alpha$-galactosidase A activity: A 14-year follow-up without enzyme replacement therapy. Nephrology (Carlton). 2016 Jul;21 Suppl 1:579. https://doi.org/10.1111/ nep.12771 PMID:26971403

11. Ersözlü $S$, Desnick RJ, Huynh-Do U, Canaan-Kühl S, Barbey F, Genitsch $V$, et al. Long-term Outcomes of Kidney Transplantation in Fabry Disease. Transplantation. 2018 Nov; 102(11):192433. https://doi.org/10.1097/ TP.0000000000002252 PMID:29688992

12. Ravarotto $V$, Simioni $F, C a-$ rraro G, Bertoldi G, Pagnin E, Calò LA. Oxidative Stress and Cardiovascular-Renal Damage in Fabry Disease: Is There Room for a Pathophysiological Involvement? J Clin Med. 2018 Nov;7(11):E409. https:// doi.org/10.3390/jcm7110409 PMID:30400144

13. Merscher S, Fornoni A. Podocyte pathology and nephropathy sphingolipids in glomerular diseases. Front Endocrinol (Lausanne). 2014 Jul;5:127. https://doi. org/10.3389/fendo.2014.00127 PMID:25126087

14. McCloskey S, Brennan P, Sayer JA. Variable phenotypic presentations of renal involvement in Fabry disease: a case series. F1000 Res. 2018 Mar;7:356. https://doi.org/10.12688/ f1000research.13708.1 PMID:29770213

15. Alfano G, Ganda N, Cerami C, Mori G, Fontana F, Cappelli G. [Screening Test of Fabry Disease in Patients with Renal Replacement Therapy in the City of Modena]. G Ital Nefrol. 2018 Mar;35(2):2018-vol2. PMID:29582965

16. Lenders $M$, Schmitz B, Stypmann J, Duning T, Brand SM, Kurschat $C$, et al. Renal function predicts long-term outcome on enzyme replacement therapy in patients with Fabry disease. Nephrol Dial Transplant. 2017 Dec;32(12):2090-7. PMID:27679524 
17. Del Pino MD, Ortiz A, Torra R, Hernández D. PrEFiNe Plan: strategic plan for Fabry diseases in Nephrology. Nefrologia. 2016 Jul-Aug;36(4):376-80. https://doi.org/10.1016/j. nefro.2016.03.009 PMID:27118193

18. Skrunes R, Svarstad E, Kampevold Larsen K, Leh S, Tøndel C. Reaccumulation of globotriaosylceramide in podocytes after agalsidase dose reduction in young Fabry patients. Nephrol Dial Transplant. 2017 May;32(5):807-13. https:// doi.org/10.1093/ndt/gfw094
PMID:27190352

19. Likhitsup A, Helzberg JH, Alba

LM, Larkin MK, Cummings

LS, Island ER, et al. Persistent Alpha-galactosidase A Deficiency After Simultaneous Liver-kidney Transplantation in a Patient With Fabry Disease. Transplantation. 2018 Aug;102(8):e361. https://doi.org/10.1097/ TP.0000000000002257 PMID:29688998

20. Yılmaz M, Uçar SK, Asçı G, Canda E, Tan FA, Hoscoskun C, et al. Preliminary Screening Results of Fabry Disease in Kidney Transplantation Patients: A Single-
Center Study. Transplant Proc. 2017 Apr;49(3):420-4. https:// doi.org/10.1016/j.transproceed.2017.01.025 PMID:28340804

21. Hopkin RJ, Cabrera G, Charrow J, Lemay R, Martins AM, Mauer $M$, et al. Risk factors for severe clinical events in male and female patients with Fabry disease treated with agalsidase beta enzyme replacement therapy: Data from the Fabry Registry. Mol Genet Metab. 2016 Sep;119(1-2):151-9. https://doi.org/10.1016/j. ymgme.2016.06.007 PMID:27510433 